\title{
Binary systems: implications for outflows \& periodicities relevant to masers
}

\author{
Nishant K. Singh ${ }^{1,2}$ and Avinash A. Deshpande ${ }^{1}$ \\ ${ }^{1}$ Raman Research Institute, C. V. Raman Avenue, Sadashivanagar, Bangalore 560080, India \\ emails: nishant@rri.res.in, desh@rri.res.in \\ ${ }^{2}$ Joint Astronomy Programme, Indian Institute of Science, Bangalore 560 012, India
}

\begin{abstract}
Bipolar molecular outflows have been observed and studied extensively in the past, but some recent observations of periodic variations in maser intensity pose new challenges. Even quasi-periodic maser flares have been observed and reported in the literature. Motivated by these data, we have tried to study situations in binary systems with specific attention to the two observed features, i.e., the bipolar flows and the variabilities in the maser intensity. We have studied the evolution of spherically symmetric wind from one of the bodies in the binary system, in the plane of the binary. Our approach includes the analytical study of rotating flows with numerical computation of streamlines of fluid particles using PLUTO code. We present the results of our findings assuming simple configurations, and discuss the implications.
\end{abstract}

Keywords. masers, radio lines: general, (stars:) binaries: general, stars: winds, outflows

\section{Introduction}

Bipolar outflows are ubiquitious in nature and presumably thought to be associated with star forming regions in molecular clouds. Since the discovery of these bipolar outflows (Snell et al. (1980)) various attempts have been made to understand the physical nature of these phenomena. Such outflows are generally believed to occur around young stellar objects (YSOs) and are thought to have intimate relationship with the process of star formation and early stage of stellar evolution (see reviews Snell (1983), Lada (1985), Bachiller (1996)). Unanimous view about the process of star formation and its early evolution is yet to emerge which in turn, inevitably, makes it difficult to have a clear understanding of the nature of these bipolar outflows. Observations of periodic/quasi-periodic variations in maser intensity from such regions add further complications (Goedhart, Gaylard \& van der Walt (2004), Goedhart et al. (2005), Goedhart et al. (2009), van der Walt, Goedhart \& Gaylard (2009), Szymczak et al. (2011), Araya et al. (2010)).

Periodicities observed in the maser light curves are one of the most challenging, poorly understood, features. In the present work, we have tried to demonstrate that a binary system, consisting of a star and another gravitating object, could be a potential candidate to explain together both of the following observed features of the maser sources, the bipolarity and the periodicity in the intensity variations.

\section{The Model and Simulations}

Consider a binary system consisting of two bodies, $S$ and $P$, which are rotating around their common center of mass, $O$, in a plane. Let $\bar{X} \bar{Y} \bar{Z}$ be the inertial (fixed) coordinate frame in which the two bodies lie in the $\bar{X} \bar{Y}$-plane with angular velocity vector in $\bar{Z}$-direction. Let $X Y Z$ be the rotating (corotating) coordinate frame which rotates with an angular velocity same as that of the two bodies in the binary system and therefore 


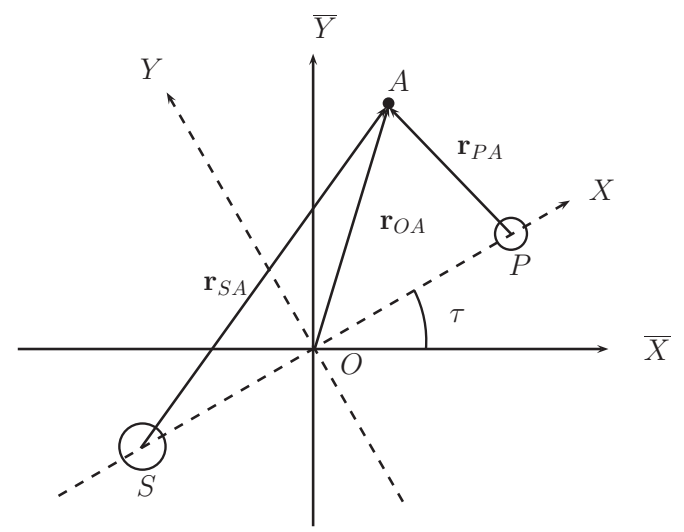

Figure 1. The inertial $(\overline{X Y})$ and rotating $(X Y)$ coordinate frames are shown in the plane of a binary. The center of mass of the two bodies labelled as $S$ and $P$ is the origin of both the coordinate frames and is denoted by $O$.

both the bodies appear to be at rest in this frame. The origins of both the coordinate frames coincide and are taken to be at the center of mass, $O$, of the binary system. The units of various quantities are chosen such that the properties of the system depend only on a single parameter. Let the total mass $(\mathcal{M})$ of the primaries $(S$ and $P$ ) be the unit of mass; the distance between them $(\mathcal{D})$ be the unit of distance; and the unit of time be chosen in such a way that the angular speed of the primaries, denoted by $\Omega$, be unity. Let $\xi$ be the mass of $P$, thus mass of $S$ is $(1-\xi)$. In the rotating reference frame, with positive $X$ in the direction of the body $P$, the coordinates of $P$ and $S$ will be $(1-\xi, 0)$ and $(-\xi, 0)$ respectively. If $(X, Y, 0)$ be the coordinate of an arbitrary point $A$, then from Figure 1,

$$
\mathrm{r}_{S A}=\left|\mathbf{r}_{S A}\right|=\sqrt{(X+\xi)^{2}+Y^{2}} ; \quad \mathrm{r}_{P A}=\left|\mathbf{r}_{P A}\right|=\sqrt{(X-(1-\xi))^{2}+Y^{2}}
$$

The gravitational potential in the corotating frame at the point $A$ may be written as,

$$
\Phi=-\frac{(1-\xi)}{\mathrm{r}_{S A}}-\frac{\xi}{\mathrm{r}_{P A}}
$$

Let us assume that one of the bodies, say $S$, has a spherically symmetric wind very near to its upper atmosphere, whereas the other body, $P$, is interacting only gravitationally. If $\boldsymbol{v}(\boldsymbol{X}, \tau)$ be the fluid velocity of the wind in the rotating frame then we may write the Euler equations in rotating frame for steady flow, with $p$ and $\rho$ as the fluid pressure and density respectively, as,

$$
(\boldsymbol{v} \cdot \nabla) \boldsymbol{v}=-\frac{\nabla p}{\rho}-\nabla \Phi-\hat{\boldsymbol{\Omega}} \times(\hat{\boldsymbol{\Omega}} \times \boldsymbol{X})-2 \hat{\boldsymbol{\Omega}} \times \boldsymbol{v}
$$

where $(\boldsymbol{X}, \tau) \equiv(X, Y, Z, \tau)$ and $\hat{\boldsymbol{\Omega}}\left(=\hat{\boldsymbol{e}}_{Z}\right.$, which is the unit vector along $\left.Z \equiv \bar{Z}\right)$ is the angular velocity of the corotating frame relative to the inertial frame.

It can be shown that,

$$
(\boldsymbol{v} \cdot \boldsymbol{\nabla}) \mathcal{B}=0
$$

where

$$
\mathcal{B}=\left(\frac{1}{2} v^{2}+\int \frac{d p}{\rho}+\Phi_{\text {eff }}\right) ; \quad \Phi_{\text {eff }}=\Phi-\frac{1}{2}|\hat{\boldsymbol{\Omega}} \times \boldsymbol{X}|^{2}
$$

Therefore the quantity, $\mathcal{B}$, is constant along a particular streamline for steady flows, although it could be a different constant for different streamlines. Noting the fact that 
the particle paths and streamlines are the same for steady flows, we can see that $\mathcal{B}$ remains the same for a particular fluid element as it moves along a particular streamline. Thus, for a particular streamline, we may write

$$
\mathcal{B}=\frac{1}{2} v^{2}+\left(\frac{\gamma}{\gamma-1}\right) R T+\Phi_{\text {eff }}=\text { constant }=C
$$

where we have used adiabatic equation of state and note that the term $\int d p / \rho$ appearing in equation 2.5 may be replaced by specific enthalpy $(w)$ for isentropic evolution of fluid element. $\gamma$ is ratio of specific heats at constant pressure and constant volume, $R$ is the gas constant and $T$ is the temperature. As the terms $v^{2} / 2$ and $\{\gamma /(\gamma-1)\} R T$ in equation 2.6 cannot be negative, we infer from equation 2.6 that the motion of a fluid element, and hence the corresponding streamline, is restricted to the region where $\Phi_{\text {eff }}<C$.

\subsection{Numerical simulations using PLUTO code}

To study how the spherically symmetric wind from the body $S$ flows in the presence of another gravitating body $P$ in a binary system, we use PLUTO code. The details of the code may be found in Mignone et al. (2007) (and references therein, and code at http://plutocode.ph.unito.it/). We use the hydrodynamic module of the code and solve the equations in three-dimensional cartesian geometry. We adapt the code in the corotating frame of the binary in which the two bodies, $S$ and $P$, appear to be at rest, by adding the necessary body-forces, namely, coriolis and centrifugal, to the equation of motion. To understand the evolution of the wind in the plane of the binary, we start with a high-pressure circularly symmetric region at the location of $S$ and the fluid pressure outside this region being very small, the fluid particles experience force which is pointed radially outward from the location of $S$. We have performed simulations for various initial conditions (by chosing different values for high/low pressure/density regions) and for different values of the parameter $\xi$. Also, the flow need not be steady. Our simulations are done in two ways: (a) we set the initial conditions and study the flow. In this case the matter eventually flows out of the computational domain as there is no supply of matter from the location of the body $S$, and the code stops; (b) Having set the initial conditions we may supply the matter at some arbitrary time intervals. In this case, as the matter is not completely depleted out of the computational domain, the code runs for longer time. How quickly the matter is depleted out of the domain depends on the initial condition. Thus, we may study the average properties of the wind flow (i.e. density, pressure etc.) by plotting density/pressure maps at different times.

\subsection{Results from PLUTO simulations and discussion}

We choose to present the result by demonstrating the evolution of density maps with time as seen in the corotating frame which is the rest frame of the binary system. This choice of plotting the density map seems relevant, as ultimately we will be interested in knowing the distribution of matter in space and its evolution in time to identify the regions which could potentially be maser emitting spots, particularly due to relatively high concentration of matter. From various panels in Figure 2, we see that the isotropy of the wind is broken very near to the binary system, as desired for density modulation seen for a fixed line-of-sight of an inertial observer. Although it is known that the orbits of a test-body are chaotic in the binary system, the problem known as the restricted three body problem (Poincaré (1890)), it is remarkable to note that the third test-body being replaced by the wind (continuous matter) evolves in a similar fashion for various initial conditions and for different values of $\xi$ as seen from simulations (e. g., the average property of the wind, say, density, seems to evolve in a definitive way). Figure 2 is the 

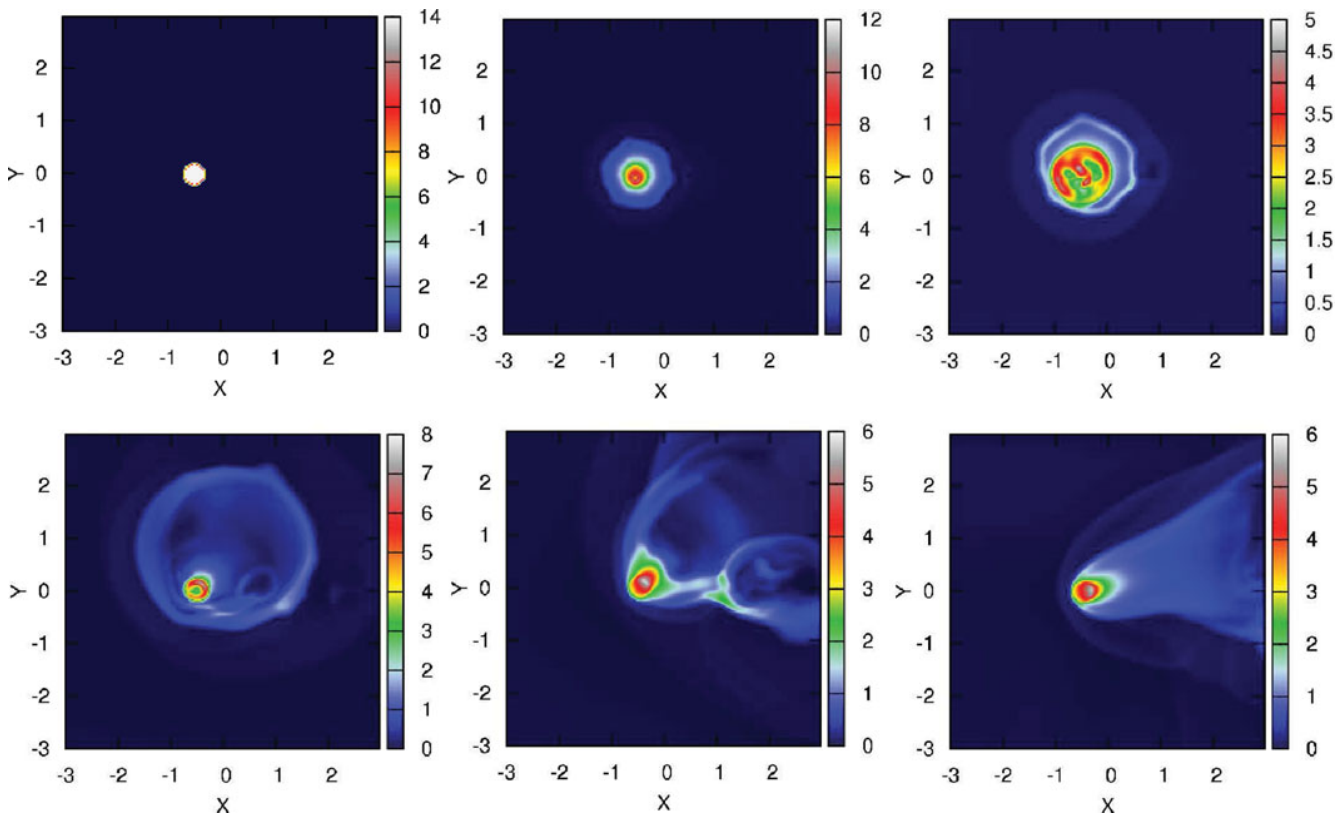

Figure 2. Snap-shots of density maps, as seen from the corotating frame (for $\xi=0.5$ and with arbitrary supply of matter at $S)$, where the bodies $S$ and $P$ are at $(-0.5,0)$ and $(0.5,0)$, respectively. Time increases from upper-left (initial time) to lower-right.

result of one of many simulations performed to study this problem. The anisotropy of the wind may be understood to be due to the shapes of the isocontours of the effective potential $\left(\Phi_{\text {eff }}\right)$ which has five Lagrange-points near to the binary, and these isocontours tend to become Keplerian far away from the binary system. Hence one may expect that the outflowing matter, which need not escape, settles into Keplerian orbits depending on their initial velocities. These results prompt us to imagine that on an average, much of the matter spirals outward with certain pitch-angle, which depends on the intial conditions, and tends to settle in Keplerian orbits far away from the binary system, forming a torus skirting the spiral pattern.

\subsection{Results of variability (light-curve) simulation}

We simulate the situation discussed at the end of last subsection and present the results by showing light-curves of maser intensities from different locations as seen along a fixed sight-line of an inertial observer. As it should, the modulations in the maser intensity will depend on the inclination of the binary system with respect to the sky-plane, and so we show our results for an arbitrarily chosen inclination angle. Columns of negligible gradients in the sight-line velocity component with adequate concentration of matter are the most preferred sites of maser emission.

\section{Summary and Conclusions}

We have investigated the flow of the wind from one of the bodies in a binary system, and tried to understand the plausible mechanisms for modulations in the maser intensity. Anisotropies seen in the rotating frame will have the desired character of bipolar flows and also will appear to be periodic (with binary-period) to an inertial observer for relevant sight-lines. Further, in our model the potential maser spots in the sky-plane do not move and the minima in intensity variation cycle repeat at regular intervals of the orbital 

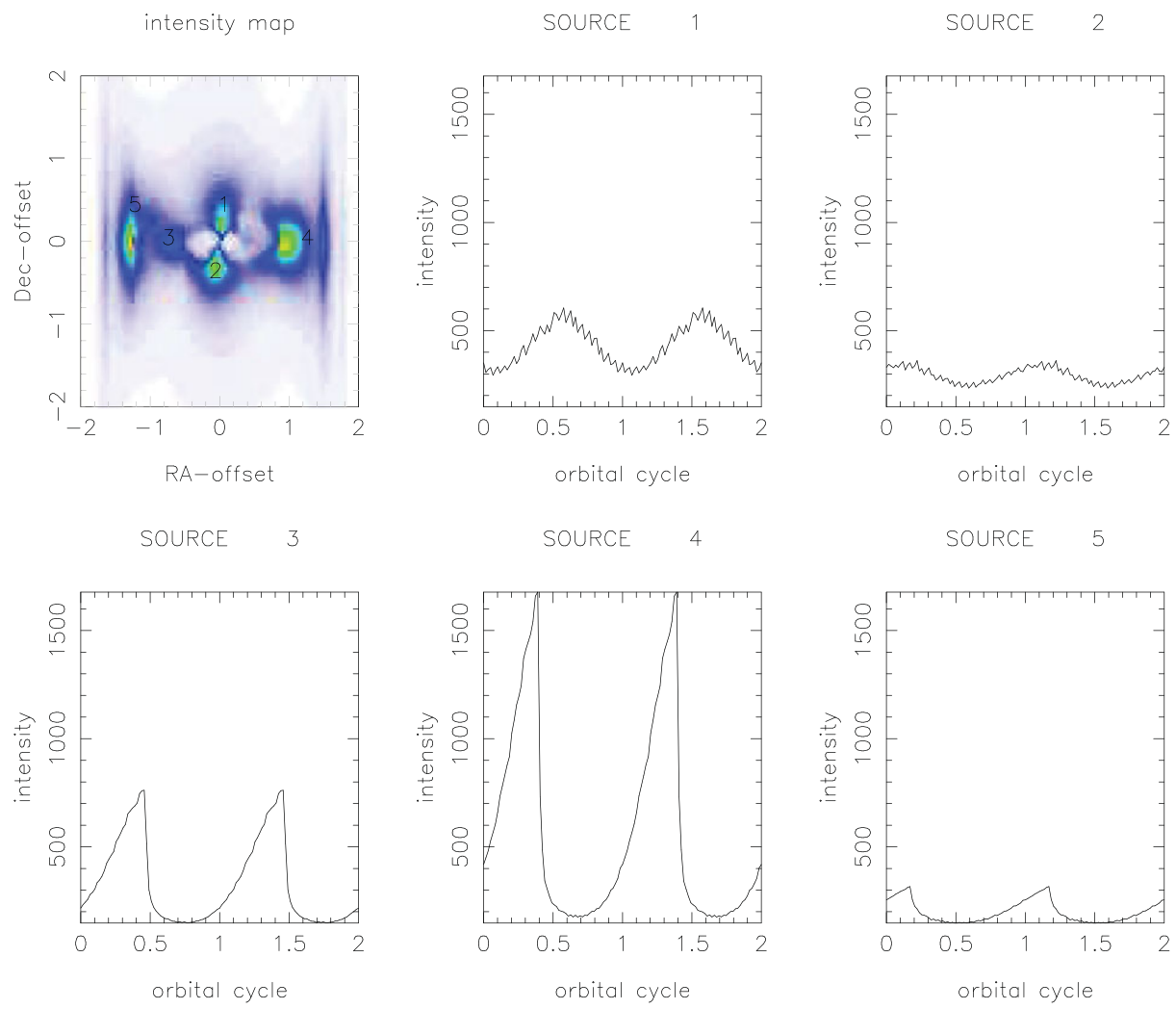

Figure 3. Light-curves (shown as line plots corresponding to the locations marked in the color panel, and over two orbital cycles) that an inertial observer would see, as monitored from our simulation of maser emission from flows in binary systems.

period. These characteristics are naturally produced in our model wherein the variabilities observed in the maser intensity is due to the density modulation resulting from the flow in the binary as seen from the simulations.

Acknowledgments: We thank the IAU, Roy Booth and other symposium organizers for providing the financial support to one of us (NKS).

\section{References}

Araya, E. D., Hofner, P., Goss, W. M., Kurtz, S., Richards, A. M. S., Linz, H., Olmi, L., \& Sewilo, M., 2010, ApJ, 717, L133

Bachiller, R., 1996, ARA\&A, 34, 115

Goedhart, S., Gaylard, M. J., \& van der Walt, D. J., 2004, MNRAS, 355, 553

Goedhart, S., Minier, V., Gaylard, M. J., \& van der Walt, D. J., 2005, MNRAS, 356, 839

Goedhart, S., Langa, M. C., Gaylard, M. J., \& van der Walt, D. J., 2009, MNRAS, 398, 995

Lada, C. J., 1985, ARA\&A, 23, 267

Mignone, A., Bodo, G., Massaglia, S., Matsakos, T., Tesileanu, O., Zanni, C., \& Ferrari, A., 2007, ApJS, 170, 228

Poincaré, H., 1890, Acta Math., 13, 1-270

Snell, R. L., Loren, R. B., \& Plambeck, R. L., 1980, ApJ, 239, L17

Snell, R. L., 1983, RMAA, 7, 79

Szymczak, M., Wolak, P., Bartkiewicz, A., \& van Langevelde, H. J., 2011, A\&SA, 531, L3

van der Walt, D. J., Goedhart, S., \& Gaylard, M. J., 2009, MNRAS, 398, 961 